\title{
Firing Squads and Fine-Tuning: Sober on the Design Argument Jonathan Weisberg
}

\begin{abstract}
Elliott Sober has recently argued that the cosmological design argument is unsound, since our observation of cosmic fine-tuning is subject to an observation selection effect (OSE). I argue that this view commits Sober to rejecting patently correct design inferences in more mundane scenarios. I show that Sober's view, that there are OSEs in those mundane cases, rests on a confusion about what information an agent ought to treat as background when evaluating likelihoods. Applying this analysis to the design argument shows that our observation of fine-tuning is not rendered uninformative by an OSE.

1 Design and the Anthropic Objection

2 Previous responses to the Anthropic Objection

3 Variations: experimental squads and survivor reunions

4 Why there is no OSE in firing squad cases

5 Application to the design argument
\end{abstract}

\section{Design and the Anthropic Objection}

Cosmological arguments from design claim that certain finely tuned features of our universe provide evidence for the existence of a cosmic designer. An example of such fine-tuning, due to McMullin ([1993]), comes from the relative strengths of the fundamental physical forces. Had the strong nuclear force been as little as 5\% weaker relative to the other forces, helium would never have formed, and our universe would contain nothing but hydrogen. Intelligent life would not have evolved. The constants of our universe are thus carefully balanced so as to allow for the existence of intelligent life, and that balance would be highly unlikely to arise as a matter of chance. Intelligent Design theorists are those who think that these considerations suggest the existence of a superintelligent designer.

Recently, Elliott Sober ([2004]) has revived an old objection to this way of thinking, which I'll call the Anthropic Objection. Although I join Sober in rejecting the design hypothesis, I find this objection unconvincing. Sober's anthropic response to the design argument rests on a confusion about what 
information an agent ought to treat as background when evaluating likelihoods. As a result, his objection commits him to rejecting patently correct design inferences in more mundane cases. If the design argument is to be rejected, I argue, we will need something better than the anthropic considerations Sober raises. ${ }^{1}$

Before we consider the Anthropic Objection, we need a clear formulation of the design argument on the table. Sober focuses on the formulation of the argument that he thinks shows the most promise: the likelihood argument. In brief this version of the argument says that, given the empirical evidence, the design hypothesis has a higher likelihood than its alternatives, and thus the evidence favours design over other hypotheses. Here 'likelihood' is used in its technical sense - the likelihood of a proposition $H$ relative to $E$ is $p(E \mid H)$. So this formulation of the design argument relies on a general principle about likelihoods:

The Likelihood Principle: If $p\left(E \mid H_{\alpha}\right)>p\left(E \mid H_{\beta}\right)$ then E favours $\mathrm{H}_{\alpha}$ over $H_{\beta}$.

Since Sober prefers to treat the design argument on objective grounds, the probabilities here, and throughout our discussion, are to be interpreted as objective chances rather than subjective credences. The likelihood version of the design argument is then

(1) If $p\left(E \mid H_{\alpha}\right)>p\left(E \mid H_{\beta}\right)$ then $E$ favours $H_{\alpha}$ over $H_{\beta}$.

(2) $p\left(E \mid H_{D}\right)>p\left(E \mid H_{\mathrm{C}}\right)$.

(3) So $E$ favours $H_{D}$ over $H_{C}$.

Here $H_{D}$ is the hypothesis that an intelligent designer created the phenomena described in $E$, and $H_{C}$ is the hypothesis that $E$ came about by chance. $E$ may describe any example of fine-tuning such as the one above, usually some fortuitous balance in the physical constants or initial conditions of the universe.

Sober's Anthropic Objection to the design argument is centered around the famous Weak Anthropic Principle (WAP):

Weak Anthropic Principle: What we can expect to observe must be restricted by the conditions necessary for our presence as observers.

The idea behind WAP is that certain kinds of observations could never be made because the nature of the environment to be observed rules out the possibility of an observer being there to observe it. An empty universe, for

1 Sober also offers a second, original objection to the design argument. But responding to that objection raises an entirely different range of issues from those relevant to the Anthropic Objection, so I discuss only the Anthropic Objection here. 
example, could not be observed since it has no inhabitants, so observations of empty universes are ruled out in accordance with WAP.

WAP is an innocuous enough claim on its own, but Sober argues that it is importantly related to the notion of an observation selection effect (OSE). Roughly, an OSE is a bias introduced into collected evidence by the method of collection. Sober illustrates with the following example from Eddington ([1939]). Suppose that we are trying to get an idea of how big the fish in a given lake are, so we go out and catch 50 fish from the lake. As it happens, all the fish we catch are 10 or more inches long, so we conclude that our evidence favours the hypothesis that all the fish are at least 10 inches. That is, we define

$E$ : All the fish we caught were longer than 10 inches.

$H_{x}: x$ of the fish in the lake are $10+$ inches.

and argue that the evidence favours $H_{1.0}$ over all other $H_{x}$ since $p\left(E \mid H_{1.0}\right)>$ $p\left(E \mid H_{x}\right)$ for $0 \leq x<1$. All is fine and good, until we realize that 10 inches is the width of the smallest hole in the net. No wonder we caught only fish $10+$ inches in length; if there were smaller ones they slipped through the holes. Thus our evidence is subject to an OSE: a bias introduced by the method of collection.

To account for an OSE when reasoning with likelihoods, we must treat the fact of the OSE as a background assumption and evaluate hypotheses in conjunction with it. That is, we look at each $p\left(E \mid H_{x} \wedge\right.$ OSE) rather than each $p\left(E \mid H_{x}\right)$. Since the OSE in Eddington's example entails that any fish caught will be $10+$ inches, it entails $E$, so that $p\left(E \mid H_{x} \wedge\right.$ OSE $)=1$ for all $0 \leq x$ $\leq 1$, which undermines the claim that $E$ favours $H_{1.0} \cdot{ }^{2}$ Thus the misleading effect of an OSE can be avoided once we are aware of its presence.

What is the connection between OSEs and WAP? Putting the two ideas together, Sober argues that the very nature of our existence as observers introduces a bias into our evidence: because we cannot observe environments in which observers cannot exist, our evidence is biased towards observations of observable environments and is thus subject to an OSE. Our nature as sentient observers introduces a sort of 'cosmological' OSE, like the holes in the fishing net. And according to Sober, the cosmological OSE (COSE) undermines the reasoning in the design argument, just as the OSE in the fishing scenario undermines the reasoning there. Having realized that we

2 Strictly speaking the OSE does not entail $E$ for a couple of reasons. First, there are improbable possibilities, e.g. a short fish getting twisted in the net. For simplicity, let us just suppose that these possibilities have been eliminated. Second, the OSE ensures only that all the fish we catch will be $10+$ inches, though we might not catch exactly 50 fish. But let us assume we know ahead of time that we will catch exactly 50 fish. 
are subject to COSE we must take it into account when evaluating likelihoods; that is, we must compare $p\left(E \mid H_{D} \wedge\right.$ COSE) with $p\left(E \mid H_{C} \wedge\right.$ COSE) instead of comparing $p\left(E \mid H_{D}\right)$ with $p\left(E \mid H_{C}\right)$. Making the appropriate substitution for premise (2) of the design argument gives us

(2) $)^{\prime} p\left(E \mid H_{D} \wedge \mathrm{COSE}\right)>p\left(E \mid H_{C} \wedge \mathrm{COSE}\right)$.

And, says Sober, since the evidence is that the universe is finely tuned for the existence of intelligent life, this observation is inevitable. Sentient beings could not exist if the universe were not fine-tuned. Thus $(2)^{\prime}$ is false because COSE entails $E$, so that $p\left(E \mid H_{D} \wedge\right.$ COSE $)=p\left(E \mid H_{C} \wedge\right.$ COSE $)=1$. In summary, Sober's Anthropic Objection is that we must reject premise (2) in favour of $(2)^{\prime}$, which he thinks is false.

\section{Previous responses to the Anthropic Objection}

Previous replies to the Anthropic Objection (Leslie [1989]; Swinburne [1990]; Van Inwagen [1993]) have denied that (2)' is false and have drawn on intuitive analogies for support. Sober discusses Swinburne's presentation of a nice example from Leslie. Suppose that you are to be executed by a firing squad of 12 members, all excellent marksmen firing 12 rounds apiece. The blindfold is tied and 144 shots ring out, but you are still alive. Does your survival favour the hypothesis that the marksmen intended to miss over the hypothesis that it was a freak occurrence? Or is there an OSE at work since you could not have observed that you did not survive? The intuitive answer, of course, is that your survival is very strong evidence that you survived by design. Never mind that you could not have observed your own death, what matters is that you observed your remarkable survival! And if you can make the design inference in this case, despite the presence of an OSE, you ought to be able to make the same inference in the cosmological design argument.

Sober deals with Leslie's case by arguing that our intuition there can be explained without appeal to likelihoods. According to Sober, the prisoner in Leslie's case is right to conclude that design is the better hypothesis, though her reasoning should be understood in terms of a conditionalization argument, as follows:

(1) $p\left(H_{D} \mid E\right)>p\left(H_{C} \mid E\right)$.

(2) $E$.

(3) Therefore $p^{\prime}\left(H_{D}\right)>p^{\prime}\left(H_{C}\right){ }^{3}$

3 Here $p^{\prime}$ is the chance function after the squad fires. 
That is, the probability that the marksmen intended to miss conditional on the prisoner's survival is greater than the conditional probability that they missed by accident, and, since the prisoner did in fact survive, conditionalization tells us that design is more probable than chance. But this conditionalization argument is different from the following likelihood argument:

(1) $p\left(E \mid H_{D}\right)>p\left(E \mid H_{C}\right)$.

(2) $E$.

(3) Therefore the evidence favours $H_{D}$ over $H_{C}$.

According to Sober the conditionalization argument is sound and accounts for the prisoner's correct conclusion that the marksmen intended to miss, whereas the likelihood argument is unsound, since its first premise should be replaced by the claim that $p\left(E \mid H_{D} \wedge \mathrm{OSE}\right)>p\left(E \mid H_{C} \wedge \mathrm{OSE}\right)$, which is false. Thus Sober recovers our intuitions about Leslie's case while maintaining that an analogy from firing squads to cosmic design is inappropriate.

A question that immediately strikes one is: why should a conditionalization argument be available to the prisoner but not to the design theorist? Because the prisoner is entitled to $p\left(H_{D} \mid E\right)>p\left(H_{C} \mid E\right)$ whereas the design theorist is not. According to Sober, there is a fundamental disanalogy between humdrum design inferences and the cosmic inferences defended by Intelligent Design theorists. In mundane cases such as Leslie's firing squad, our claim that $p\left(H_{D} \mid E\right)>p\left(H_{C} \mid E\right)$ is justified by frequency data and our general knowledge of human behaviour. But no such theory or data are available to ground the analogous claim in the case of the design argument. Though the prisoner can, for example, note the ratio of observed firing squad survivals that are due to each of design and chance, and she can use these quantities to estimate $p\left(H_{D} \mid E\right)$ and $p\left(H_{C} \mid E\right)$, the Intelligent Design theorist cannot do this because she has no data about what fraction of finely tuned universes were created by intelligent designers.

\section{Variations: experimental squads and survivor reunions}

The trouble with Sober's way of handling Leslie's case is that it is easy to construct variants where the prisoner does not have a conditionalization argument available but still ought to think her survival favours design. Frank Arntzenius offers the following example. ${ }^{4}$ Suppose, as before, that you are standing for execution before a firing squad. But this time you know that your would-be executioners are using experimental guns, guns that have never been tested. You also know that these guns come in two

4 Personal communication. 
models, Type A and Type B. While you have no experience with either sort of gun in the field, you do have intimate knowledge of the guns' inner workings and a good physical theory that allows you to draw a reliable conclusion about how accurate each type of gun will be. Based on this information, you conclude that Type A guns are vastly more accurate than Type B guns. The moment of truth comes, 144 shots ring out, and you stand unscathed. What conclusion should you draw: is the firing squad using Type A guns or Type B guns, or does your inability to observe your own execution prevent you from drawing either conclusion?

Clearly the prisoner ought to conclude that her survival favours the Type A hypothesis, since it better accounts for the remarkable fact that all 144 shots missed. But Sober will have to say that she should not draw this conclusion. Arntzenius's variant is designed so that the prisoner does not have any frequency data from which to conclude that $p(A \mid E)>p(B \mid E)$, since she has no experience with firing squads using the experimental guns. She does, however, have enough data to conclude that $p(E \mid A)>p(E \mid B)$, since her physical theory and knowledge about the guns' workings allow her to make predictions about her chances of survival. The intuition being pumped is that this is enough for her to conclude that her survival is evidence that the firing squad is using Type A guns. But if the prisoner cannot draw this conclusion via a conditionalization argument or, as Sober must say, via a likelihood argument, how are we to account for the apparent reasonableness of her conclusion?

Sober does not address such variations explicitly, but he does try to explain away our temptation to think that the prisoner in Leslie's case is entitled to a likelihood argument for $H_{D}$. He argues that the temptation arises from a confusion between a first-person and third-person perspective of the prisoner's situation:

To assess the claim that the prisoner has made a mistake [in declining to favour $H_{D}$ ], it is useful to compare the prisoner's reasoning with that of a bystander who witnesses the prisoner survive the firing squad... I suggest that part of the intuitive attractiveness of the claim that the prisoner has made a mistake derives from a shift between the prisoner's point of view and the bystander's. The bystander is right to use $p\left(E \mid H_{\mathrm{D}}\right)>p\left(E \mid H_{C}\right)$ to interpret his observations; however, the prisoner has no business using $p\left(E \mid H_{D}\right)>p\left(E \mid H_{C}\right)$ to interpret his observations since he, the prisoner, is subject to an OSE. The prisoner needs to replace $p\left(E \mid H_{D}\right)>p\left(E \mid H_{C}\right)$ with $p\left(E \mid H_{D} \wedge\right.$ OSE $)=p\left(E \mid H_{C} \wedge\right.$ OSE $)$. [Sober 2004, p. 138]

Can our intuitions about Arntzenius's variant be similarly dispatched? Can Sober say that the bystander should favour the Type A hypothesis whereas the prisoner should not? I do not think so. For consider what happens when

5 I am translating Sober's notation into my own. 
the survivor and the bystander meet: according to Sober they will disagree about which guns were used and they are right to do so. We might not think much of this, since cases of disagreement about which hypothesis ought to be accepted often arise when agents have different evidence. But in this example, we can stipulate that the prisoner and her observer have identical epistemic starting points, and Sober will still have to say that they must draw radically different conclusions. So this move has the unhappy consequence that rational agents must disagree, even though they have identical epistemic starting points and identical epistemic inputs. ${ }^{6}$

To bolster his position, Sober argues that his opposition is also committed to an unattractive consequence. He asks us to consider a case where a thousand firing squad survivors are assembled to pool their evidence:

\begin{abstract}
Suppose that a firing squad always subjects its victims to the same probabilistic process, which has the result that the prisoner either survives or is killed. A thousand prisoners who have one by one each survived the firing squad are assembled and are asked to pool their knowledge and estimate the value of an unknown probability. What is the probability that a prisoner will survive if the firing squad fires?... Those who believe that the single prisoner has evidence about his firing squad's intentions are obliged to conclude that the best estimate in this new problem is that the probability is unity. [Sober 2004, p. 139]
\end{abstract}

But this argument contains a non-sequitur. Sober assumes that if a prisoner's own survival is evidence for inaccuracy, then her observation of any prisoner's survival must be evidence for inaccuracy. But why should we think that? Whether or not a given prisoner's survival is evidence for inaccuracy depends on how that prisoner is selected for observation. If she is chosen randomly then her survival can provide such evidence. If she is selected for observation at a survivors' reunion, however, she certainly cannot provide any evidence. Similarly, if an entire sample of prisoners is selected for observation at a survivors' reunion, then observing a $100 \%$ survival rate in the sample provides no evidence about the squad's accuracy. We are free to say, and ought to say, that our prisoner gains no information about the squad's accuracy upon meeting other survivors at the reunion, since her sample is subject to an

6 Sober has suggested in personal correspondence that this disagreement between prisoner and bystander may not be a problem: upon meeting the bystander, the prisoner will take on the bystander's testimony as evidence and conclude that the squad intended to miss. I see two problems with this move. First, before meeting, the prisoner and bystander still draw inconsistent conclusions from identical epistemic resources. That is damning enough. But second, what 'testimony' can the prisoner take on as evidence of design? That the bystander was there to see the whole thing happen? The prisoner might be aware throughout the ordeal that the bystander is overseeing the execution, in which case she already knows this fact and gains no information from the meeting. And it will not do to respond that, in cases where the prisoner is aware of the bystander all along, she should favour design upon surviving. Then whether the prisoner should favour design will depend on whether she is aware of a third-person observer. 
OSE. The ball is then in Sober's court, since he is left to explain the disagreement between the prisoner and bystander in Arntzenius's firing squad case.

\section{Why there is no OSE in firing squad cases}

So far my response to the Anthropic Objection has rested on an analogy with firing squad scenarios. I have been arguing that one's survival in firing squad cases is not nullified by an OSE, so the same ought to hold for the design argument. But it will be useful to take the analysis further. For once we understand why the alleged OSE in firing squad scenarios is bogus, we can apply a similar analysis to the design argument. The case against Sober will then be maximally strong. We will not need to rely on an analogy in order to argue that the COSE does not undermine the evidence; we will be able to show why it is a mistake to say that it does.

Start with Leslie's firing squad. There the alleged OSE is the fact that you cannot observe your own non-survival. Sober says that this undermines your observation of survival as evidence because it entails the evidence. But no such entailment holds. What information does the prisoner have about her methods of data collection that guarantees her observation of her own survival? None, to be sure. After all, if she had such information she would not have to worry about being shot! Rather, what she does know is that if she observes anything at all, it will be her survival. And this in no way entails that she will survive. Sober's contention that there is an OSE in firing squad cases rests on a confusion between two propositions:

$S$ : I will observe that I survive.

$S^{\prime}$ : If I observe whether I survive, I will observe that I survive. ${ }^{7}$

Although $S$ certainly entails the evidence, it is clearly inappropriate for the prisoner to use $S$ as a background assumption when evaluating the evidential import of her survival. And $S^{\prime}$, though it may be a legitimate background assumption, does not entail that the prisoner will survive.

Responding to this analysis, Sober has suggested ${ }^{8}$ that, even once the $S / S^{\prime}$ distinction is drawn, we still find that the prisoner is subject to an OSE. For even though she does not know before the execution that she will survive, once the squad misses and she decides to determine whether or not she has survived, it is inevitable that she will find that she has. 'Inevitable' how? In that her discovery is entailed by something she already knows: when the

7 The conditional here is material, since what you know is just that you will not observe whether you have survived and find that you have not. Of course, the converse conditional is true as well.

8 Personal correspondence. 
prisoner decides to check whether she has survived, she knows things such as 'I am now checking whether I survived.' And, since these facts entail the evidence, they make the evidence equiprobable regardless of the squad's intentions, thereby undermining her ability to discriminate between $H_{D}$ and $H_{C}$.

But if the prisoner already has information that entails her survival, all this tells us is that we have been evaluating the wrong evidence. Rather than evaluating her observation that she exists, the prisoner ought to evaluate the entailing information as evidence instead. That is, let $E_{1}=$ 'I exist' and $E_{2}=$ 'I am checking whether I exist.' Sober's argument is that $E_{1}$ is undermined by $E_{2}$ since $p\left(E_{1} \mid H_{D} \wedge E_{2}\right)=p\left(E_{1} \mid H_{C} \wedge E_{2}\right)$. But this just suggests that she consider the evidential import of $E_{2}$ instead. So the prisoner has the following likelihood argument available to her:

(1) $p\left(E_{2} \mid H_{D}\right)>p\left(E_{2} \mid H_{C}\right)$.

(2) $E_{2}$.

(3) Therefore $E_{2}$ favours $H_{D}$ over $H_{C}$.

The moral is this: the fact of her survival is going to enter the prisoner's store of evidence at some point, whether through direct observation or via some entailing fact, and when it does she will have a likelihood argument available to her that favours $H_{D}$ over $H_{C}$.

Sober also questions my $S / S^{\prime}$ analysis of firing squad scenarios on the grounds that the same analysis gets the wrong results in uncontroversial cases such as Eddington's fishing example. Here is the idea: suppose we tweak Eddington's case so that I leave my net in the lake and plan to come back and check it in two weeks. In keeping with the $S / S^{\prime}$ distinction, what I know with certainty is not that I will find a net full of fish that are all $10+$ inches, but that if I come back to check my net in two weeks, I will discover that all the fish I catch are $10+$ inches. But since this fact does not entail the observation I make upon returning (I might have died in the meantime or forgotten to come back), Sober worries that I am committed to saying there is no OSE here.

But this criticism rests on a false assumption. It assumes that, because my formulation of the OSE fails to entail the evidence, it fails as an OSE. But notice that for an OSE to render $E$ uninformative with respect to $H_{\alpha}$ and $H_{\beta}$, it need not entail $E$. Rather what is needed is just $p\left(E \mid H_{\alpha} \wedge \mathrm{OSE}\right)=p\left(E \mid H_{\beta} \wedge\right.$ OSE), so that a likelihood argument is not available for either hypothesis. Naturally, if the OSE entails $E$, then we get the special case of this where $p\left(E \mid H_{\alpha} \wedge \mathrm{OSE}\right)=p\left(E \mid H_{\beta} \wedge \mathrm{OSE}\right)=1$, but that is just one way things can go. So Sober cannot object that my formulation of the OSE in Eddington's case fails because it does not entail the evidence. What he would need to show 
instead is that the likelihoods of the relevant hypotheses differ, even when conjoined with my formulation of the OSE. And precisely the opposite is true. Given that you will observe all $10+$ inch fish if you return to check the net, the chance that you will find only $10+$ inch fish is just the chance that you will return to check your net - regardless of the makeup of the lake's population. I take it this fact is intuitive, but a formal statement and proof can be given. Let $E$ and $H_{x}$ be as in the original presentation of Eddington's case (above) and let $R \supset E$ be the material conditional 'If I return to check my net in two weeks' time, I will find only fish that are $10+$ inches.' Then for $0 \leq x \leq 1$ we have:

$$
\begin{aligned}
p\left(E \mid H_{x} \wedge[R \supset E]\right) & =\frac{p\left(\left[E \wedge\left(H_{x} \wedge \neg R\right)\right] \vee\left[E \wedge\left(H_{x} \wedge E\right)\right]\right)}{p\left(\left[H_{x} \wedge \neg R\right] \vee\left[H_{x} \wedge E\right]\right)} \\
& =\frac{p\left(E \wedge\left[H_{x} \wedge \neg R\right]\right)+p\left(E \wedge\left[H_{x} \wedge E\right]\right)}{p\left(H_{x} \wedge \neg R\right)+p\left(H_{x} \wedge E\right)} \\
& =\frac{p\left(H_{x} \wedge E\right)}{p\left(H_{x} \wedge \neg R\right)+p\left(H_{x} \wedge E\right)} \\
& =\frac{p\left(H_{x}\right) p(E)}{p\left(H_{x}\right) p(\neg R)+p\left(H_{x}\right) p(E)} \\
& =\frac{p(E)}{p(\neg R)+p(E)}
\end{aligned}
$$

Since the chance of the observation is the same no matter which $H_{x}$ we conditionalize on, its informativeness is nullified. Notice the proof assumes that $\neg R$ and $E$ are probabilistically independent of the $H_{x}$ s. The fact that an analogous assumption is not warranted in firing squad cases-since whether or not you will be around to check for your survival is probabilistically tied to hypotheses about the squad's accuracy - captures the difference between Leslie's case and the genuine OSE in Eddington's case.

\section{Application to the design argument}

All that remains is to apply this discussion to the design argument. Recall that the relevant likelihoods are $p\left(E \mid H_{D} \wedge \mathrm{COSE}\right)$ and $p\left(E \mid H_{C} \wedge \mathrm{COSE}\right)$, which Sober claims are identical since COSE entails $E$. We are now in a position to see that this view makes the same mistake as the view that the prisoner has no evidence about the squad's intentions. It confuses the proposition that we must observe fine-tuning with the proposition that we cannot observe anything else. Recall that in the firing squad case we distinguished between $S$ and $S^{\prime}$ :

$S$ : I will observe that I survive.

$S^{\prime}$ : If I observe whether I survive, I will observe that I survive. 
The mistake was in thinking that the prisoner should evaluate the likelihoods of competing hypotheses in conjunction with $S$ rather than $S^{\prime}$. So here too we must distinguish between COSE and COSE':

COSE: I will observe a finely tuned universe.

$\mathrm{COSE}^{\prime}$ : If I observe whether the universe is fine-tuned, I will find that it is.

And once the distinction is made, it is clear that WAP at best entitles us to the latter claim, $\mathrm{COSE}^{\prime}$. But $\mathrm{COSE}^{\prime}$ does not entail that we will observe finetuning since we might not have existed at all. To close the gap Sober must insist that we conjoin $\operatorname{COSE}^{\prime}$ with the fact of its antecedent; call it $A$. That is, he must argue that premise (2) of the design argument ought to be replaced by the claim $p\left(E \mid H_{D} \wedge \operatorname{COSE}^{\prime} \wedge \mathrm{A}\right)>p\left(E \mid H_{C} \wedge \operatorname{COSE}^{\prime} \wedge A\right)$, which is false since $\operatorname{COSE}^{\prime} \wedge A$ entails $E$. But the moral we have learned from firing squad scenarios is that we ought not to treat our existence as an 'inevitable' background assumption, much less the fact that we will be around to check for fine-tuning. Our existence is not inevitable and can thus be evidentially informative. Moreover, since $\operatorname{COSE}^{\prime} \wedge A$ not only entails $E$ but is equivalent to it, insisting that we treat $\operatorname{COSE}^{\prime} \wedge A$ as a background assumption amounts to treating $E$ as both the evidence and the background simultaneously. But that is like evaluating the evidential import of a coin's having landed heads while using the fact that the coin landed heads as a background assumption. Such practice renders all evidence uninformative and is surely poor methodology. ${ }^{9}$

Interestingly, even if we were to grant Sober that $A$ ought to be treated as a background assumption when evaluating $E$, his view would still be faulty. I said that WAP at best entails $\operatorname{COSE}^{\prime}$, but careful reflection shows that it doesnot even do that. WAP is the very weak claim that what we can expect to observe is restricted by the conditions necessary for a sentient observer to be present. Prima facie that looks to entail $\operatorname{COSE}^{\prime}$, but the entailment goes through only if we assume that sentience requires fine-tuning. Certainly the sort of sentience that we are does require fine-tuning because of its organic and physical basis, but if we allow that sentience can exist independently of the physical-bodiless spirits and such-then WAP will not guarantee us $\mathrm{COSE}^{\prime}$. We might have been mere Cartesian souls and found ourselves inhabiting a chaotic universe. So even if we grant Sober that both WAP and our

9 We might worry that there is still an important disanalogy between firing squads and the design argument. In firing squad cases there is a time before the execution when the prisoner does not know that she will survive, but there is no time before our inception as rational agents at which we can consider whether or not we will exist and doubt that we will. Thus the evidence used in the design argument is inevitable in a way that the prisoner's discovery of her survival is not. But this concern just points up a minor flaw of firing squad cases. To avoid it, just consider the case where it was your pregnant mother before the firing squad, rather than you. 
existence as sentient observers are inevitable, and therefore ought always be treated as background assumptions, Sober will still be wrong that our observation of fine-tuning is inevitable. ${ }^{10}$

The upshot is that Sober's anthropic critique of the design argument is seriously wanting. If he wishes to say that our existence must always be conjoined with a hypothesis whose likelihood is to be evaluated, he has yet to provide good reasons for his position, not to mention a satisfactory analysis of firing squad scenarios. Certainly there are other arguments in the area that I have not addressed (see [Bostrom 2002], for example), and it is possible that further analysis would vindicate such a view. Nevertheless, Sober's own arguments do not.

\section{Acknowledgements}

I would like to thank Frank Arntzenius, David Manley, and Chris Meacham for much helpful discussion. I am also especially indebted to Elliott Sober and an anonymous referee for their generous and illuminating comments on an earlier draft of this paper.

Department of Philosophy

Rutgers University

26 Nichol Ave.

New Brunswick, NJ 08901

USA

jweisber@rci.rutgers.edu

\section{References}

Bostrom, N. [2002]: Anthropic Bias: Observation Selection Effects in Science and Philosophy, New York: Routledge.

Eddington, A. [1939]: The Philosophy of Physical Science, Cambridge: Cambridge University Press.

Inwagen, P. V. [1993]: Metaphysics, Boulder, CO: Westview Press.

Leslie, J. [1989]: Universes, London: Routledge.

McMullin, E. [1993]: 'Indifference Principle and Anthropic Principle in Cosmology', Studies in the History and Philosophy of Science, 24, pp. 359-89.

Sober, E. [2002a]: 'Bayesianism-Its Scope and Limits', in Bayes' Theorem: Proceedings of the British Academy Press, 113, pp. 21-38.

10 It is tempting to dismiss this argument that WAP does not entail $\operatorname{COSE}^{\prime}$ on the grounds that it countenances the possibility of disreputable beings like Cartesian souls. But notice that the entire debate presupposes this possibility. We are considering the hypothesis that the universe was fine-tuned by a cosmic designer, which implies that the designer, a sentient being, managed to exist without that fine-tuning. So insofar as we are willing to take the design argument seriously at all, we must take this argument seriously too. 
Sober, E. [2002b]: 'Intelligent Design and Probability Reasoning', International Journal for the Philosophy of Religion, 52, pp. 65-80.

Sober, E. [2004]: 'The Design Argument', in W. E. Mann (ed.), Blackwell Guide to the Philosophy of Religion, Oxford: Blackwell Publishers, pp. 117-147.

Swinburne, R. [1990]: 'Arguments from the Fine-Tuning of the Universe', in J. Leslie (ed.), Physical Cosmology and Philosophy, New York: MacMillan, pp. 160-79. 